\section{Enfermedad periodontal, su terapia: un reto}

Periodontal disease, its therapy : a challenge

\title{
ARTículo Cú́ntCos
}

\author{
Luis Vidal Maita Véliz'; ; María \\ Castañeda Mosto ${ }^{2 *}$; Luis Mariano \\ Maita Castañeda y Carmen Navarro \\ Contreras ${ }^{4}$
- Departamento Académico de Estomatologí Medico Quirargico
2 Departannento Acadernico de Estomatología Pediátrica
37 Consulta privada, av. Vargas Machuca 194, Lima 18, Perta
* Facultad de Odontologia, Universidad Nacional Mayor de San Marcos, Lima, Pert. Emmail: 1maxitav@unmstr. edu.pe

\section{Resumen}

El diagnóstico y tratamiento periodontal debe ser un objetivo en el ejercicio de la Odontolología. Para lograr este propósito el paciente debe estar al tamente motivado y el odontologo realizar la etapa más importante: la terapia de mantenimiento, la cual permitirá conservar sanos los tejidos perindontales- especialmente el hueso- toda la vida, además de sus piezas dentarias.

\begin{abstract}
The diagnosis and treatmnet of the periodontal disease must be an objetive in the dental practice. To reach this purpose, the patient must have high motivation and the dentist must work in the maintenance periodontal therapy; this leads to have healthy periodontal tissue- specially the bone- for ever and the teeth also.
\end{abstract}

Palabras claves: Enfcrmedad pcriodontal, motivación, terapia periodontal de mantenimiento.

\section{Introducción}

En el ejercicio profesional diario es frecuente observar condiciones periodontales en los pacientes, en estado avanzado de destrucción de los tejidos periodontales especialmente el tejido óseo, cuyo nivel de pérdida llega al tercio apical o al âpice dental, constituyendo un estado de periodontitis avanzada de difícil tratamiento y pronóstico reservado o malo.

Se considera a las enfermedades periodontales como la causa principal de la pérdida de las piezas dentarias a partir de los 30 - 40 años en la población mundial en general.

Entonces surge la pregunta: ¿Por qué se llega a estas condiciones avanzadas de destrucción ósea con la secuela de pérdida de el mayor numero de piezas dentarias?

¿Se pudo prevenir la aparición de la enfermedad periodontal?

Según cálculos estadísticos estimados en otros países como EE.UU'. a cantidad de personas afectadas por la enfermedad periodontal estaría entre 17 a 36 millones de su población. En el caso hipotético de que cada uno de los 3,700 periodoncistas de U.S.A, tratasen 200 casos con enfermedad periodontal destructiva severa por año, colectivamente se podría tratar 740,000 casos de Periodontitis al año

E1 tiempo de tratamiento que sè necesitaría para tratar a esta población, trabajando periódicamente a un promedio de 200 pacientes por año se podría calcular que tomaría 23 años tratar a los 17 millones de pacientes y 48 arios para tratar a los 36 millones de pacientes. Además, todos estos pacientes necesitarian toda la ayuda de los dentistas de práctica general $y$ de las asistentes dentales para el control o mantenimiento posterior de la enfermedad periodontal para los años futuros ${ }^{1}$.

En nuestro medio son pocos los estudios epidemiológicos realizados sobre enfermedad periodontal ${ }^{2,3}$ y menos aûn en niños cuya etapa inicial es reversible (gingivitis) y puede ser perfectamente controlada y prevenida ${ }^{4}$.
Key words: Periodontal disease, motivation, supportive periodontal therapy.

\section{Objetivos de la terapia periodontal}

La diversidad de artículos de investigación y textos en Periodontología están de acuerdo en forma unánime que la motivación es el principal recurso para iniciar un tratamiento periodontal.

Sin embargo poco se "dice" o no se explica como lograr motivar al paciente .El proceso de motivación debe ser exitoso para que el paciente pueda detener y eliminar la enfermedad periodontal posiblemente estos sean aspectos filosóficos en la Periodoncia todavia no analizados por los inves. tigadores en el área de la motivación periodontal.

Motivación es un término amplio utilizado en psicología para comprender las condiciones o estados que activan o dan energía al organismo, que llevan. a una conducta dirigida hacia determinados objetivos.

Los motivos a impulsos, como se les llama con frecuencia, pueden ser innatos en su naturaleza, o aprendidos; 
pero, cualquiera que sea su origen, cuando son despertados, inician una actividad dirigida hacia determinadas finalidades o incentivos, que han sido relacionados con los motivos particulares comprendidos a través del aprendizaje.

El proceso de motivación no es simple. Las etapas de la motivación son:

1) Anticipación 2) Activación y dirección 3 - Conducta activa y retro alimentación de rendimiento y 4) Resultados.

Entre los lineamientos para un programa de motivación se considera las variables de la personalidad a trabajar: La autoestima y el autoconocimiento.

Es importante señalar que las técnicas motivacionales son la entrevista y la observación. En la entrevista los aspectos relevantes son las preguntas; con ellos se podrán analizar la relación odontólogo- paciente, si es activa o pasiva y si hay una mutua participación.

En la observación, juega un papel importante el análisis de habilidades en la técnica de limpieza bucal.

Las características de los pacientes para obtener un grado de salud periodontal son: la edad, el sexo, (las damas son mas preocupadas para lograr una salud bucal en general) profesión, valores, expectativas disponibilidad de tiempo para dedicar a la salud, También debemos considerar las características demográficas, culturales sociales y económicas. Es importante diferenciar entre-informar al paciente y educar al paciente.

Al final de este proceso se podrá lograr:

1) Que el paciente entenderá (como se desarrolla la Enfermedad periodontal en la boca de cada uno de ellos)

2) Que las instrucciones sobre higiene periodontal deberán ser puestas en práctica

3) Que los pacientes soliciten una atención odontológica integral.

4) Que los pacientes se controlen periódicamente su salud periodontal en función de sus necesidades individuales.

5) Que algunos pacientes evidentemente carezcan de motivación adecuada o de la habilidad o destreza necesaria para lograr un cuidado periodontal adecuado y no es candidato para posteriores tratamientos periodontales quirúrgicos, porque definitivamente se producirá la recidiva de la Enfermedad Periodontal.

Se debe recordar que en $1 \mathrm{~mm} 3$ de placa dental que pesa aproximadamente $1 \mathrm{mg}$ están presentes 108 de bacterias; es decir millones de bacterias en solo un espacio pequeñísimo.

La investigación científica en estudios experimentales en animales $y$ estudios longitudinales en humanos ${ }^{2}$, han demostrado que en la mayoría de personas, aún en niños: un tratamiento que incluya la eliminación de la placa bacteriana por medios mecánicos y químicos se logra recuperar la salud periodontal ${ }^{4}$.

Esto constituye una estrategia en el tratamiento odontológico en general y debe ser llevada a cabo en lo consultorios por los dentistas de práctica general.

Además debemos considerar que el control de la placa bacteriana y el hábito de fumar son considerados en la actualidad factores de riesgo para la periodontitis crónica.

\section{Terapia Periodontal de Mante- nimiento (TPM)}

La Academia Americana de Periodoncia define la "terapia periodontal de mantenimiento" como "una extensión de la terapia periodontal con procedimientos realizados a intervalos establecidos para ayudar al paciente periodontal en el mantenimiento de la salud bucal" .Por lo que debe ser parte integral del tratamiento de las enfermedades periodontales.

Nuestros conocimientos sobre la evolución natural de las enfermedades periodontales y la pérdida de inserción periodontal que esta conlleva justificarían las necesidades de aplicar TPM preventiva a la población general. Hoy en día poseemos numerosos estudios que avalan científicamente la necesidad de TPM preventiva. Lövdal y cols., en un estudio ya clásico, estudiaron las condiciones gingivales de un grupo de 1.428 personas durante un periodo de cinco años. Durante este tiempo los individuos recibieron instrucciones de higiene bucal y raspaje dos-cuatro veces al año. Los resultados demostraron una disminución del $50 \%$ en las pérdidas dentarias con respecto a un grupo control, mientras que las condiciones gingivales (índice de placa, índice de sangrado, profun- didad de sangrado, etc.) mejoraron en torno a un $60 \%$.

Axelsson y cols (6) publicaron un estudio longitudinal (15 años) sobre prevención de caries dental y enfermedad periodontal Los resultados demostraron una pérdida de 0,2 dientes por individuo, el número de superficies que presentaba placa era minoritario (rango 10- 20\%), los pacientes solo presentaban un $3 \%$ de unidades gingivales inflamadas y se produjo una pequeña ganancia de inserción clínica (media $0,3 \mathrm{~mm}$ ).

La mayoría de los estudios sobre la evolución natural de la enfermedad periodontal se han referido a las pérdidas dentarias. Hoy en día sabemos que estas ocurren en un grupo reducido de personas. Este hecho nos indica la importancia que para la prevención de la enfermedad periodontal y el mantenimiento en salud en estos grupos de riesgo tendría el diagnóstico precoz, lo que nos permitiria la realización de una TPM individualizada, según sus necesidades.

En los últimos 40 años son numerosas las publicaciones que, sin ser exactamente estudios sobre TPM y refiriéndose tanto a estudios clínicos prospectivos longitudinales como de estudios retrospectivos, vienen a demostrar la posibilidad de mantener la salud periodontal de nuestros pacientes durante largos periodos de tiempo.

Entre los primeros cabe destacar el trabajo de Suomi y cols, en el que compararon la evolución de 1.248 individuos que tras el tratamiento periodontal fueron divididos, de acuerdo al protocolo de mantenimiento establecido, en dos grupos.

En el primero de ellos se reforzó en la motivación para el control de la placa y profilaxis cada dos meses el primer año, cada tres el segundo y cada cuatro el tercero, mientras que el segundo solo fue explorado una vez al año sin recibir ningún tratamiento. Los resultados mostraron una pérdida de inserción clínica de $0,03 \mathrm{~mm}$ en el primer grupo y de 0,1 mm en el segundo.

Axelson y Lindhe (7) trataron periodontalmente a 77 pacientes que presentaban periodontitis avanzada y que posteriormente fueron divididos en dos grupos. Cincuenta y dos de ellos siguieron un estricto programa de TPM durante seis años, mientras que el resto fueron nuevamente remitidos a su odontólogo para realizar 
"cuidados dentales tradicionales". Al final del estudio se evidencio la estabilidad de los resultados obtenidos después del tratamiento en el grupo de pacientes bajo TPM, mientras que el otro grupo presentó elevados índices de placa y gingivales, asi como signos de recidiva de la enfermedad, demostrando la importancia que la TPM y el refuerzo en el control de placa tionen en el éxito a largo plazo de los tratamientos periodontales

De los estudios retrospectivos, debemos destacar por el número de pacientes y la amplitud del periodo estudiado el trabajo de Hirschfeld y Wasserman. Estos autores estudiaron a 600 pacientes periodontales de los cuales, en el momento del diagnóstico el $77 \%$ presentaba periodontitis avanzada, un $16 \%$ periodontitis moderada y un $7 \%$ periodontitis leve. Fueron tratados y mantenidos durante un período de 15 a 50 años (media de 22 años). Los resultados mostraron pérdidas del $7,1 \%$ de sus dientes por motivos periodontales. Asimismo, mostraron pérdidas de solo el $33 \%$ de los que aquellos dientes considerados en el estadio inicial de mal pronostico (furcas III, movilidad $2 / 2,+$ bolsas de $7 / 10 \mathrm{~mm}$.)

Publicaciones recientes han centrado sus esfuerzos en estudiar la eficacia de la TPM en colectivos concretos de pacientes. Rosling y $\mathrm{col}^{8}$ estudiaron la evolución de 225 pacientes periodontales tras tratamiento periodontal no quirúrgico y TPM. Estos pacientes fueron divididos en dos grupos: pacientes categorizados como "normales" y pacientes de "alta susceptibilidad". Los resultados, tras 12 años de estudio, demostraron la estabilidad de los resultados obtenidos durante la fase activa del tratamiento en la mayoría de los pacientes "normales" (pérdida de inserción clínica media de $0,45 \mathrm{~mm}$ ), con muy pocas pérdidas dentales $(0,3$ dientes por paciente). Sin embargo, los pacientes de alta susceptibilidad experimentaron una pérdida de inserción significativamente mayor (pérdida de $0,8 \mathrm{~mm})$ y más pérdida dentaria $(1,9$ dientes por paciente). No obstante, concluyeron que en el $95 \%$ de los pacientes "normales" y el $80 \%$ de los pacientes "de alto riesgo" se mantenúan los niveles óseos y la inserción periodontal estables.

Presentamos 02 casos cuyo tratamiento periodontal consistió en el primero solo a base de fisioterapia periodontal es decir se le enseñó la correcta higiene periodontal por medio del cepillado sulcular o la técnica de Bass, utilizando un cepillo de 2 hileras y además hilo dental sin cera. El resultado como se aprecia es excelente.

\section{Caso 1}

Es un paciente de 22 años que asiste al consultorio por problemas de sangrado gingival desde hace 6 meses. Ha recibido tratamiento dental previo sin ningún resultado favorable.

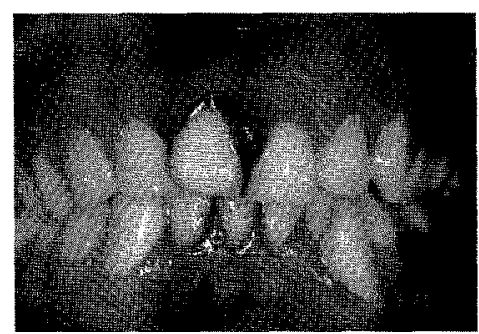

Fig.1: El paciente en su visita inicial el 25 -mayo-2000 con las alteraciones gingivales y sangrado expontáneo.

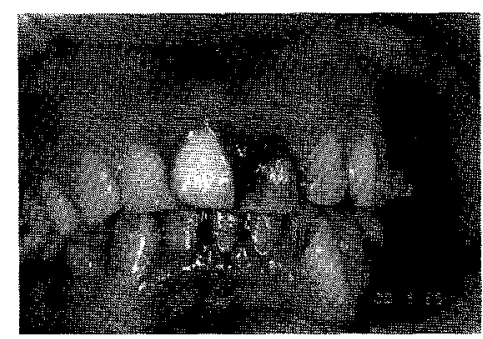

Fig. 2: Obsérvese la placa dental y el apiñamiento inferior del lado izquierdo.

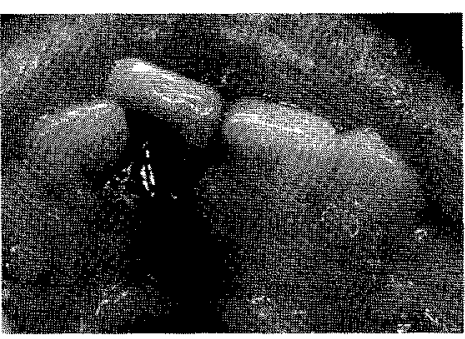

Fig. 3: En la cara palatina de los dientes anterosuperiores se aprecian el sangrado profuso y expontáneo.

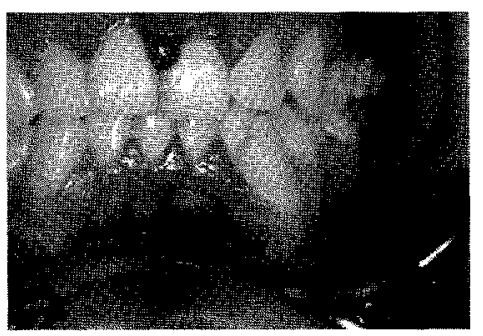

Fig. 4: Nueve días después de iniciado l cepillado periodontal, los cambios gingivales son favorables (3-junio- 2000)

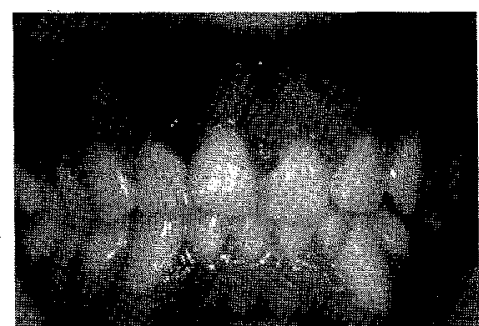

Fig. 5: 13 días despues del cepillado periodontal, en menos de dos semanas, el paciente ha recuperado mas del $50 \%$ de su salud periodontal (7-junio-2000)

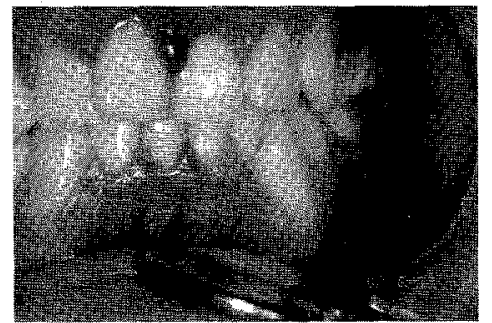

Fig. 6: 23 dias después del tratamiento periodontal el paciente ha recuperado cerca del $80 \%$ de salud periodontal ( 17 junio- 2000)

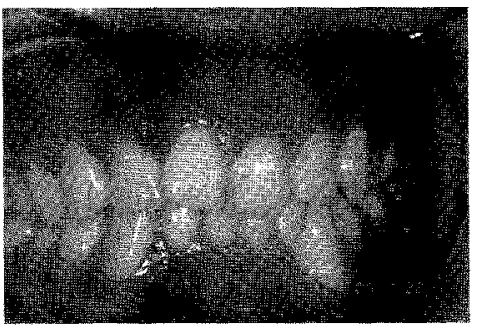

Fig. 7: 58 días (menos de 02 meses) el paciente ha recuperado su salud periodontal al $100 \%(22-$ julio 2002$)$

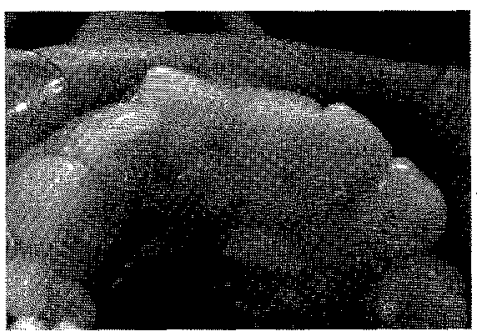

Fig. 8: Excelente higiene periodontal, despues de 1 año 2 meses. Comparece con la fig. 3, (26-julio 2001)

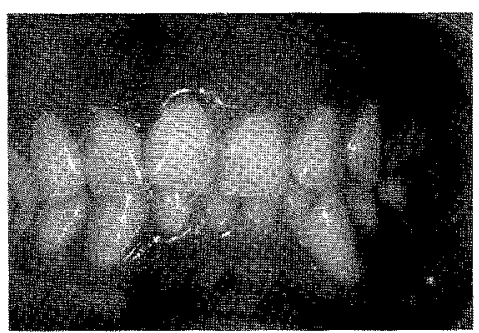

Fig 9: Tratamiento exitoso al $100 \%$ después de 1 año 2 meses. 


\section{Caso 2}

Paciente de 16 años que acude al consultorio dental por presentar sangrado gingival, molestias bucales diversas, dificultad para comer y al cepillado. Recibió tratamiento periodontal a base de fisioterapia periodontal, raspaje y alisado radicular.

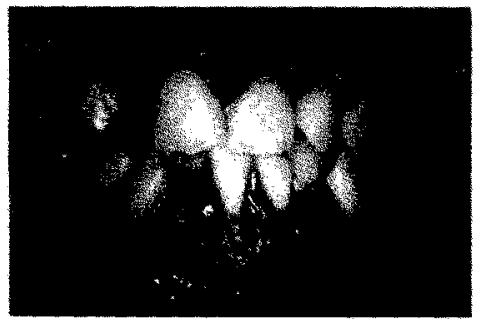

Fig. 1: Estado inicial como se presenta el paciente el (7-agosto-1997). Los cambios patológicos periodontales son tan evidentes en ambos maxilares.

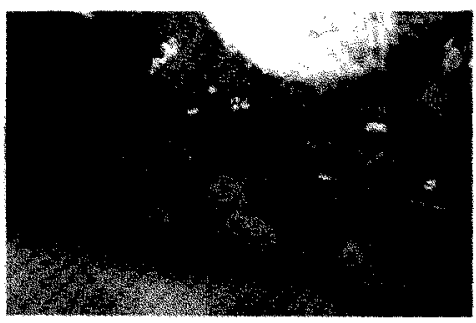

Fig. 2: Observe los crecimientos del tejido gingival en los dientes anteroinferiores (12-agosto-1997).

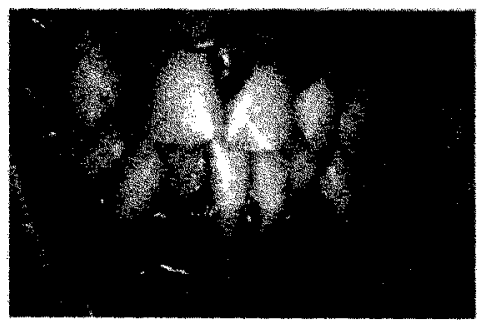

Fig. 3: Después de 4 meses, ha mejorado la salud periodontal a pesar d e la falta de motivación por parte del paciente (13diciembre-1997).

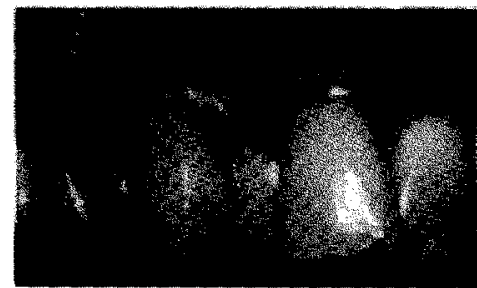

Fig. 4: El paciente vuelve a preocuparse por su salud periodontal después de 6 años y sigue rigmosamente el protocolo de tratamiento periodontal para logra los resultados que se aprecian. (15-enero $2003)$.

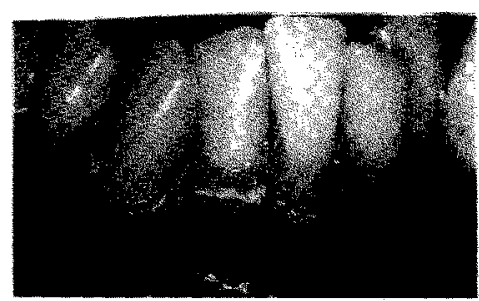

Fig. 5: Resultados obtentios mediante higiene periodontal, raspaje y alisado radicular (15- enero-2003)

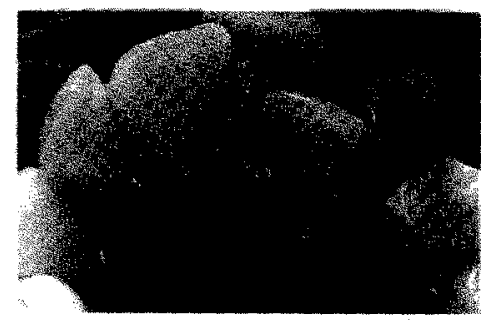

Fig. 6: El apiñamiento dental no fue obstáculo para lograr la salud periodontal al 100\% (15-enero- 2003)

\section{Referencias}

1. American Academy of Periodontology: Periodontal disease Management A, Conference for the Dental Team, Boston, Massachusetts, July 1993 U.S.A.
2. Maita Véliz, Luis: Diagnostico Precoz de la Enfermedad Periodontal en niños y adolescentes mediante el índice de sangrado- papilar de Mühlemann (PBI).Avances en Periodoncia. Vol 4 No1-Mayo 1992. Madrid, España.

3. Maita Véliz, Luis; Castañeda Mosto Maria: Estudio Longitudinal periodontal en una población no controlada después de nueve años. Rev. Scienta Omni, U.N.MS.M, Vol 1, N ol, pág. 40-45. Lima 1997.

4. Navarro, Contreras Camen: Efectividad de la clorhexidina y de los medios mecánicos para reducir gingivitis en nifnos de 10 a 12 años. Tesis de Bachiller. Facultad de Odontología. Universidad San Martin de Porras, 2005.

5. Lindhe Jan, T. karring; N. P. Lang. Clinical Periodontology and Implant Dentistry. Blackwell Munksgaard, Publishing Company (fourth edition) 2003.

6. Axelsson P, Lindhe I, Nystron B: On the prevention of caries and periodontal disease. Result of a 15 years longindinal study in adults.]. Clin Periodontol, $1991 ; 17 \div 182-189$.

7. Axelsson P, Lindhe J: The significance of maintenance care in the treatment of periodontal disease J. Clin. Periondol $1981 ; 8: 281-294$

8. Rosling B, Serino G. Helström MK Sokransky SS, Lindhe J. Longitudinal periodontal tissue alterations during supportive therapy. Finding from subjects whit normal and.high susceptibility to periodontal disease. J. Clin. Periodontol, $2001 ; 28: 241-249$. 